\title{
Higiene e saúde bucal em idosos na atenção primária: uma revisão sistemática
}

\author{
Hygiene and oral health in elderly in primary care: a systematic review
}

Higiene y salud bucal en ancianos en atención primaria: una revisión sistemática

Ana dos Santos Araújo ${ }^{1 *}$, Marilda Andrade ${ }^{1}$, Fabiana de Melo Amaral Gonçalves Pinto1.

\section{RESUMO}

Objetivo: Identificar a relação entre higiene e saúde bucal em idosos saudáveis e com síndromes demenciais na atenção primária, destacando o efeito de ações educativas sobre o comportamento de idosos, cuidadores e profissionais. Métodos: Trata-se de uma revisão sistemática de literatura, utilizando-se as bases de dados BBO, Medline e Lilacs, com corte temporal de cinco anos (2015-2019). A estruturação da pesquisa deu-se através do acrônimo PICO, utilizando-se os descritores (P) Aged and Primary Health Care and (I) Oral Hygiene and $(\mathrm{C})$ não se aplica $(\mathrm{O})$ Oral Health. Utilizaram-se estudos referentes à população idosa, com foco em higiene bucal, e excluíram-se os que abordavam percepções profissionais sem foco na higiene bucal. Resultados: Incluíram-se seis artigos, sendo quatro em inglês e dois em português. Os artigos abordaram a relação entre higiene e saúde bucal em idosos saudáveis e naqueles com alguma debilidade, como Doença de Parkinson e Doença de Alzheimer, discutindo, também, a importância de ações educativas para profissionais e cuidadores, assim como a falta de orientações em alguns casos. Conclusão: Vislumbram-se novas amostras para que sejam elaboradas políticas e programas eficientes e direcionados à promoção da saúde bucal em idosos.

Palavras-chave: Idoso, Atenção primária à saúde, Higiene bucal, Saúde bucal.

\section{ABSTRACT}

Objective: To identify the relationship between oral hygiene and health of the elderly, healthy or with dementia syndromes, in primary health care, highlighting the effect of educational actions on the behavior of the elderly, caregivers and professionals. Methods: This is a systematic literature review, using Lilacs, Medline and BBO databases, with a 05-year time cut (2015-2019). The research structuring occurred through the acronym PICO, using the descriptors (P) Aged and Primary Health Care and (I) Oral Hygiene and (C) not applicable (O) Oral Health. The sample included studies referring to the elderly population, focused on oral hygiene, and excluded studies addressing only health professionals' perspective, not focusing on oral health. Results: Six articles were obtained, four in English and two in Portuguese. The articles addressed the relationship between oral hygiene and health in the healthy elderly and those with some type of disease, such as Parkinson's Disease and Alzheimer's Disease, also discussing the importance of educational actions for health professionals and caregivers, in addition to the lack of guidance in some cases. Conclusion: New samples are necessary to elaborate efficient policies and programs directed to the promotion of oral health in elderly people.

Key words: Aged, Primary health care, Oral hygiene, Oral health.

\section{RESUMEN}

Objetivo: Identificar la relación entre higiene y salud bucal en ancianos sanos y con síndromes de demencia en atención primaria, destacando el efecto de las acciones educativas en el comportamiento de los ancianos, cuidadores y profesionales. Métodos: Esta es una revisión sistemática de la literatura, utilizando las bases

${ }^{1}$ Universidade Federal Fluminense (UFF), Niterói - RJ. *E-mail: ana.araujoasa@gmail.com 
de datos Lilacs, Medline y BBO, con un corte temporal de 05 años (2015-2019). La estructuración de la investigación ocurrió a través del acrónimo PICO, utilizándose los descriptores (P) Aged and Primary Health Care and (I) Oral Hygiene and (C) no se aplica (O) Oral Health. Se incluyeron los estudios que abordaron la población anciana, con enfoque en la higiene bucal, y se excluyeron los estudios que abordaron las percepciones profesionales, sin enfoque en la higiene bucal. Resultados: Se utilizaron seis artículos, cuatro en inglés y dos en portugués. Los artículos abordaron la relación entre la higiene y la salud bucal en ancianos sanos y aquellos con alguna debilidad, como la enfermedad de Parkinson y la enfermedad de Alzheimer, y también discutieron la importancia de las acciones educativas para profesionales y cuidadores, además de la falta de orientaciones en algunos casos. Conclusión: Se vislumbran nuevas amuestras para desarrollar políticas y programas eficientes destinados a promover la salud bucal en los ancianos.

Palabras clave: Anciano, Atención primaria de salud, Higiene bucal, Salud bucal.

\section{INTRODUÇÃO}

Discutir a realidade demográfica da população idosa, analisar suas particularidades e seu nível de acesso à saúde bucal tornam-se cada vez mais urgentes, uma vez que problemas como cáries e doenças periodontais são muito comuns e podem acarretar em uma deterioração das estruturas dentárias, o que, por sua vez, pode levar à extração parcial ou total dos dentes, situação que compromete a qualidade de vida do idoso. Desta forma, é primordial assegurar ao idoso o acesso aos cuidados bucais preventivos e, em último caso, curativos.

Em tempos passados, o acesso odontológico sofria com as inúmeras barreiras existentes na assistência à saúde: as técnicas e recursos eram obsoletos e os profissionais acabavam realizando procedimentos hoje tão simples de forma brusca e deterioradora. A idade senil tem suas particularidades, e nem sempre a saúde, a higiene e a condição do idoso responderão no mesmo nível, principalmente aqueles pacientes em situações demenciais, portadores de doenças crônicas, dentre outros, comprometendo sua higiene bucal ou tornandoos dependentes ou com sua capacidade funcional debilitada (SIMÕES ACA e CARVALHO DM, 2011; BARBE AG, et al., 2017).

A associação entre ações de saúde bucal pelos profissionais e cuidadores e o autocuidado pelo paciente tem impacto positivo na vida e saúde deste grupo quando realizada de forma contínua e correta, destacandose, aqui, a importância do dentista e demais profissionais envolvidos neste cuidado, e do fortalecimento e ensejo das políticas em saúde nesta temática, já que os estudos atuais apontam para a longevidade da população. O modelo assistencial aos idosos precisa ser vigorado periodicamente, e a antiga atenção odontológica radical deve ser desmistificada, na busca para oferecer a este grupo qualidade de vida, oportunidade de autocuidado e prevenção de problemas bucais, os quais, muitas vezes, podem se agravar e desencadear riscos sistêmicos (SIMÕES ACA e CARVALHO DM, 2011).

A concepção da atenção primária veio para fortalecer a organização do sistema de saúde de indivíduos e comunidade, de forma regionalizada, ininterrupta e ordenada, através de movimentos profiláticos e terapêuticos. A década de 1970, período em que a situação da saúde no Brasil encontrava-se precária, foi demarcada pela Reforma Sanitária, que refletia sobre melhores ofertas de saúde para a sociedade. Esta reforma possibilitou a $8^{\text {a }}$ Conferência Nacional de Saúde em 1986, o que favoreceu, através da Constituição Federal (CF) de 1988, a oficialização do Sistema único de Saúde (SUS), que estabelece o princípio da universalidade do direito à saúde a todos. Em 1996, o Ministério da Saúde (MS) criou a Norma Operacional Básica (NOB) - SUS 01/96, que promoveu a descentralização do modelo assistencial. Em 2001, o MS criou a Estratégia da Saúde da Família (ESF) e a Norma Operacional de Assistência à Saúde (NOAS) - SUS 01/2001, garantindo aos municípios assistência a nível regional e o mínimo de atenção básica aos usuários. Em 2000, regulamentou-se, em portaria, a inclusão da saúde bucal na ESF, e, em 2004, foi vigorada a Política Nacional de Saúde Bucal (PNSB), conhecida como Brasil Sorridente (SOUZA AC, et al., 2012).

Embora a assistência tenha aprimorado, este progresso deve ser aliado à realidade de cada grupo, tendo em vista que muitas unidades de atenção ainda carecem de ações educativas, de acesso eficaz a todos, 
assim como uma atenção holística aos pacientes que se encontram com suas funções fragilizadas ou inúteis. Uma má condição bucal pode desencadear candidíase, xerostomia, dentre outras, que, por sua vez, podem disseminar-se a ponto de degradarem as peças da arcada dentária, atrapalharem o processo de mastigação, provocarem um hálito desconfortável, diminuição da saliva, o que leva ao crescimento de bactérias nocivas ao homem, propiciando infecções mais graves (SILVA HPR, et al., 2017).

O processo de envelhecimento é algo comum e inevitável a todo ser humano, cujas alterações fisiológicas naturais podem desencadear condições muitas vezes incontornáveis, e, assim, os cuidados aos idosos, devem perpassar uma simples assistência para seu processo de melhoria e cura (MOREIRA RM, et al., 2013). A população idosa brasileira vem aumentando de forma considerável. Dessa forma, é inevitável o surgimento de desafios e investigações para a oferta de uma qualidade de vida e melhores condições de saúde bucal, tendo em vista que ainda existem muitos problemas relacionados a uma higiene oral adequada e eficaz (MOREIRA RM, et al., 2013).

O trabalho integrado entre as equipes de odontologia, enfermagem e demais equipes de saúde no âmbito da Atenção Primária à Saúde visa, sobretudo, à prevenção de riscos e danos à saúde do paciente, ainda mais quando se remete ao idoso, grupo diferenciado pela degradação da sua resistência física, de acessibilidade, condições mentais, emocionais e por aspectos relacionados a fatores econômicos, sociais e culturais, grau de instrução precário, muitas vezes barreiras para o ingresso dos idosos à atenção bucal (MOREIRA RM, et al., 2013).

A partir do exposto, foi possível perceber que o enfermeiro, o dentista e os demais membros da equipe de saúde atuam cada um na sua competência, mas de maneira direcionada para o cuidado integral, e, ao perceber a necessidade de cada usuário, cada profissional atua ou designa o cuidado ao respectivo profissional sem deixar que os cuidados se dissociem, mas que sempre se integrem (COLUSSI CF e CALVO MCM, 2011).

O enfermeiro trabalha o cuidado, e, dentro desse cuidado, está a saúde bucal. Na visita domiciliar, analisase a presença de alguma alteração, que será tratada e orientada pelo profissional que realiza a visita, ou sinalizada para aquele que ficará responsável pela intervenção, sendo, neste caso, o dentista. Muitas orientações são dadas pelo próprio enfermeiro, visto que são informações básicas e de aprendizado durante o Curso de Graduação de Enfermagem, como os cuidados básicos de higiene bucal, e até mesmo a detecção de um sangramento de gengiva, relatos de dor pelo usuário, dentre outros (MINISTÉRIO DA SAÚDE, 2008). A atenção primária precisa caminhar atenta junto aos profissionais, instituições e níveis de instâncias em longo prazo para que ocorrências e problemas ainda presentes sobre a temática da cavidade bucal, assim como os riscos atinentes à esta cavidade sejam amenizados ou erradicados em nosso país e nas instituições.

Antigamente, havia a retirada total ou parcial das peças dentárias, o que não permitia a continuidade de cuidados e informações sobre os meios de prevenção, o que repercutia na propagação de outras doenças. Segundo Silva, o envelhecimento, em seu processo de transição demográfico, estava relacionado aos surtos das doenças e aos meios obtidos para a prevenção. Essa condição proporcionou queda de mortes por infecção, porém, o fato de os idosos viverem por mais tempo fez com que eles ficassem mais expostos às doenças incuráveis, que degradariam muito mais a sua saúde. Os estudos apontam sobre a reavaliação de assistência pelos sistemas de saúde na criação de novas demandas para intervirem nos problemas atuais do grupo (DUTRA CESV e SANCHEZ HF, 2015; SILVA HPR, et al., 2017).

Neste sentido, a pesquisa em questão justifica-se por evidenciar a importância do entrelaçamento das competências de enfermeiros e odontólogos no cuidado e tratamento da saúde oral de idosos que procuram a atenção primária à saúde. A equipe de enfermagem e de odontólogos na ESF são elos importantes e indissociáveis na manutenção e promoção do cuidado na saúde oral.

Com base neste cenário complexo em que se estabelece a saúde bucal do paciente idoso no âmbito da atenção primária à saúde, a pesquisa buscou identificar a relação entre higiene e saúde bucal em idosos saudáveis e com síndromes demenciais na Atenção Primária, destacando o efeito de ações educativas sobre o comportamento de idosos, cuidadores e profissionais. 


\section{MÉTODOS}

Optou-se por um estudo nos moldes de revisão sistemática acerca da prevenção de doenças por meio da saúde bucal na atenção primária. Utilizou-se a estratégia PICO (Paciente, Intervenção, Comparação e Resultados) para direcionamento da pesquisa, elegendo-se os seguintes descritores para o acrônimo (P) Aged and Primary Health Care and (I) Oral Hygiene and (C) não se aplica (O) Oral Health, tendo como questão clínica: os idosos com acesso à educação em saúde na atenção primária têm maiores chances de preservar a saúde bucal? Para tanto, foram utilizadas as bases de dados Bibliografia Brasileira de Odontologia (BBO), Literatura Latino Americana e do Caribe em Ciências da Saúde (LILACS) e Medline.

Os descritores utilizados foram levantados pelo DeCS da BVS (Biblioteca Virtual de Saúde), onde foi realizada a busca, sendo eles: idoso (aged/anciano), atenção primária à saúde (primaryhealthcare/atención primaria de salud), higiene bucal (oral hygiene/higiene bucal) e saúde bucal (oral health/salud bucal). Para realizar a combinação dos descritores, foi utilizado o operador Booleano AND. Esta revisão é composta de seis etapas: Escolha da temática, seguido da questão norteadora com conteúdo que seja relevante dentro da sociedade, escolha das bases de dados para seleção dos estudos, determinação dos critérios que serão pertinentes ou não para a pesquisa, conteúdo sólido e concreto dos estudos escolhidos nas bases de dados, finalização do estudo embasado em dados científicos (BARBE AG, et al., 2017).

Para eleger os textos de interesse, foi realizada uma leitura prévia, onde foram selecionados os textos com informações proveitosas para o trabalho. Depois, procedeu-se a uma leitura seletiva dos resumos para escolher as publicações que tratavam de assuntos relevantes ao tema. Ao perceber que os resumos não deixavam claro o objetivo da pesquisa, optou-se pela leitura dos artigos na íntegra.

Os critérios de inclusão definidos para a seleção dos artigos foram: Artigos publicados em português, inglês ou espanhol; que tratassem de população idosa, publicados nos últimos cinco anos (2015-2019). Os critérios de exclusão foram: artigos que tratassem apenas de percepção de profissionais, que não abordassem higiene como complemento do assunto principal (saúde bucal). O levantamento de títulos ocorreu no mês de abril de 2019.

Após a realização da busca, os artigos foram exportados no formato RIS e agrupados no gerenciador de referências Mendeley para análise de resumos e exclusão de duplicatas de forma automatizada. A seleção dos artigos obedeceu a seguinte ordem: leitura de resumos e títulos para verificar se as pesquisas contemplavam o assunto principal e, posteriormente, leitura completa das obras, para confirmação da validade de seu conteúdo dentro do proposto, realizada por duas revisoras de forma independente.

\section{RESULTADOS}

Na base de dados Bibliografia Brasileira de Odontologia, combinaram-se os descritores da seguinte forma: idoso AND atenção primária à saúde AND higiene bucal AND saúde bucal, obtendo-se 25 estudos. Ao aplicar o filtro "ano de publicação", foram encontrados três artigos. Destes, dois foram excluídos por não abordarem a temática de interesse. Dessa forma, foi selecionado apenas um estudo desta base de dados.

Considerando-se a Medline, foram encontrados 14 artigos, dos quais, após leitura dos títulos e resumos, excluíram-se 10 por não apresentarem relação com o objeto de estudo, restando quatro para leitura do texto completo. Já na base de dados LILACS, a combinação dos descritores idoso AND atenção primária à saúde AND higiene bucal resultou em dois artigos, sendo ambos excluídos por discutirem temáticas diferentes. Nesta mesma base dados, a combinação dos descritores idoso AND atenção primária à saúde AND saúde bucal resultou em 31 artigos. Destes, foram excluídos 29 artigos que não abordavam a temática, restando dois para leitura do texto na íntegra.

Como havia um estudo repetido entre as bases de dados Bibliografia Brasileira de Odontologia e LILACS, foi mantido o que estava indexado na LILACS. Com isso, foram selecionados seis artigos para leitura do texto completo, sendo todos eles incluídos na amostra para integrar esta revisão sistemática. A Figura 1 mostra o fluxograma que descreve as etapas de seleção dos estudos. 
Figura 1 - Fluxograma com as etapas de seleção dos artigos.
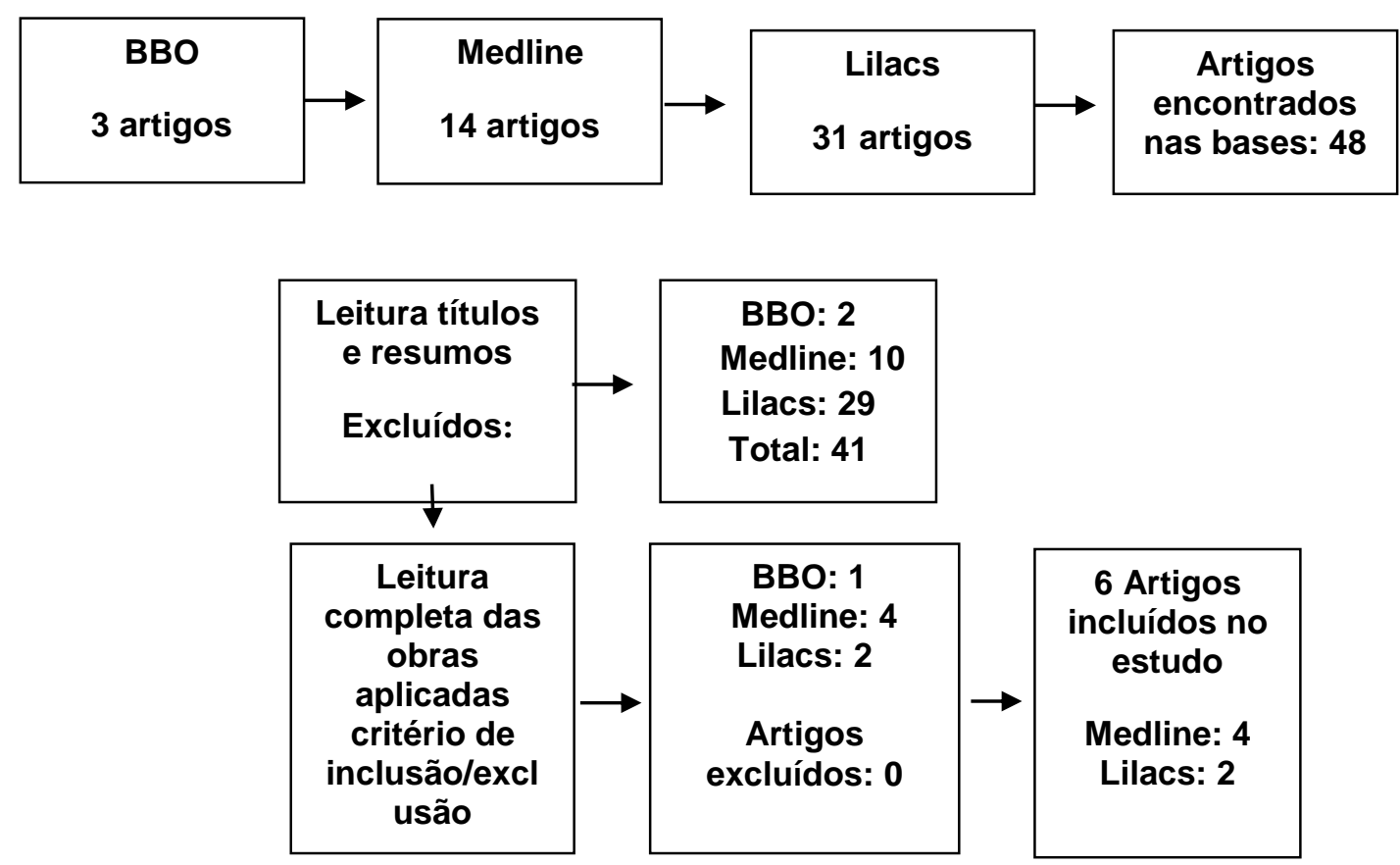

Fonte: Araújo AS, et al., 2019.

Após a seleção dos estudos, eles foram organizados de acordo com os autores, ano de publicação, objetivo e resultados. Dos seis artigos, quatro foram publicados em inglês, enquanto dois foram publicados em português. Com relação ao ano de publicação, 2015 apresenta três $(50,00 \%)$ artigos publicados, o ano de 2016 , dois (33,33\%), e 2017, um (16,67\%). O Quadro 1 mostra os seis artigos selecionados e organizados conforme explicitado anteriormente. 


\section{Revista Eletrônica Acervo Saúde / Electronic Journal Collection Health ｜ ISSN 2178-2091}

Quadro 1 - Artigos recuperados na revisão sistemática

\begin{tabular}{|c|c|c|c|}
\hline Autor & Ano de publicação & Objetivo & Resultado \\
\hline Barbe AG, et al. (2017) & 2017 & $\begin{array}{l}\text { Explorar a atenção odontológica autoavaliada em } \\
\text { pacientes com Parkinson, incluindo a capacidade de } \\
\text { realizar higiene bucal, auto avaliação da xerostomia, } \\
\text { problemas de baba e disfagia. }\end{array}$ & $\begin{array}{c}\text { Os participantes sofreram xerostomia (49\%), } \\
\text { baba }(70 \%) \text { e disfagia (47\%) e sofriam de uma } \\
\text { capacidade limitada de realizar higiene bucal } \\
(29 \%) .\end{array}$ \\
\hline Osmari D, et al. (2016) & 2016 & $\begin{array}{l}\text { Avaliar o comportamento dos idosos quanto aos } \\
\text { procedimentos de higiene e manutenção de próteses } \\
\text { removíveis. }\end{array}$ & $\begin{array}{c}\text { Esta população não estava ciente da importância } \\
\text { da higiene e manutenção das próteses } \\
\text { removíveis. }\end{array}$ \\
\hline Pradeep AR, et al. (2015) & 2015 & $\begin{array}{l}\text { Comparar o estado periodontal entre indivíduos com e } \\
\text { sem doença de Parkinson para determinar a influência } \\
\text { desta na doença periodontal. }\end{array}$ & $\begin{array}{l}\text { Há uma necessidade de atendimento } \\
\text { odontológico e encorajamento no uso de } \\
\text { métodos de controle de placa para pacientes com } \\
\text { Parkinson, uma vez que a patologia periodontal } \\
\text { apresentou alta prevalência mesmo nos estágios } \\
\text { iniciais da doença de Parkinson. }\end{array}$ \\
\hline Wang TF, et al. (2015) & 2015 & $\begin{array}{l}\text { Avaliar os efeitos da educação em saúde bucal para } \\
\text { cuidadores sobre o estado de saúde bucal de idosos. }\end{array}$ & $\begin{array}{l}\text { A revisão sistemática e meta-análise encontrou } \\
\text { evidências limitadas de que a educação em } \\
\text { saúde bucal para os cuidadores pode ser eficaz } \\
\text { para melhorar a saúde bucal dos idosos. }\end{array}$ \\
\hline $\begin{array}{l}\text { Warmling AMF, et al. } \\
\qquad(2016)\end{array}$ & 2016 & $\begin{array}{l}\text { Identificar estratégias utilizadas na atenção à saúde } \\
\text { bucal de idosos com doença de Alzheimer em casa. }\end{array}$ & $\begin{array}{c}\text { As estratégias empregadas estão relacionadas } \\
\text { ao grau de dependência da pessoa idosa, pois o } \\
\text { cuidador atua com base na necessidade de } \\
\text { cuidados com a saúde bucal e nas dificuldades } \\
\text { em realizar tais cuidados. }\end{array}$ \\
\hline Bizerril D, et al. (2015) & 2015 & $\begin{array}{l}\text { Identificar ações e atividades desenvolvidas pelo } \\
\text { cirurgião-dentista nas visitas domiciliares. }\end{array}$ & $\begin{array}{c}\text { O cirurgião-dentista nas visitas domiciliares } \\
\text { assume um importante papel de facilitador para o } \\
\text { bem-estar físico e social dos usuários } \\
\text { impossibilitados de ir até uma Unidade de } \\
\text { Atenção Primária à Saúde. }\end{array}$ \\
\hline
\end{tabular}

Fonte: Araújo AS, et al., 2019. 


\section{DISCUSSÃO}

Este estudo objetivou identificar a relação entre higiene e saúde bucal em idosos saudáveis e com síndromes demenciais na atenção primária à saúde. Por ser uma temática muito abrangente, este trabalho procurou selecionar artigos que abordassem o efeito de atividades educativas sobre o comportamento de idosos, cuidadores e profissionais, e aqueles que discutissem a própria relação entre a higiene bucal (auto avaliada, ou com avaliação de terceiros, quando possível) e a saúde bucal de idosos. Abrangeram-se idosos saudáveis e com síndromes demenciais pelo fato de poder mostrar a influência que tais enfermidades possuem sobre a higiene/saúde oral.

Em 2010, existiam, no Brasil, 39 idosos para cada grupo de 100 jovens, ou seja, 10 đ da população total, atingindo, em 2020, 13,7\% da população total do país (MIRANDA GMD, et al., 2016; SANTOS CS, et al., 2020). Até 2040, estima-se que haverá 153 idosos para cada 100 jovens, revelando um envelhecimento populacional, para o qual o país ainda não está bem preparado (MIRANDA GMD, et al., 2016). Em termos de saúde bucal, uma revisão de literatura revelou que, com o passar dos anos, há o surgimento de algumas dificuldades motoras, o que pode comprometer a capacidade de limpeza e higienização, que passa a ser executada por familiares, cuidadores ou profissionais de saúde (SALES MVG, et al., 2017).

Quando se trata de idosos dependentes ou semi-dependentes, incluindo aqueles que possuem algum tipo de síndrome demencial, como Doença de Alzheimer, a visita domiciliar torna-se uma estratégia eficaz para analisar seus graus de dependência e direcionar as orientações que thes serão fornecidas. Tais pacientes podem realizar sua higiene bucal de forma integral, ou contar com o auxílio total ou parcial do cuidador/profissional (BIZERRIL D, et al., 2015; WARMLING AMF, et al., 2016). De acordo com o Ministério da Saúde (2017), a Atenção Domiciliar (AD) é uma forma de atenção à saúde, em que se oferece um conjunto de ações de promoção à saúde, prevenção e tratamento de doenças e reabilitação na moradia do paciente, estando este serviço disponível no Sistema Único de Saúde (SUS). Este cuidado é realizado por diferentes equipes, dependendo da complexidade do usuário, e está ligado diretamente aos aspectos referentes à estrutura familiar, à infraestrutura do domicílio e à estrutura oferecida pelos serviços de saúde, evitando-se, desta forma, hospitalizações desnecessárias e reduzindo o risco de infecções (MINISTÉRIO DA SAÚDE, 2017).

Todavia, as visitas domiciliares não são realidade em muitos locais do país, e, assim, procedimentos como aplicação tópica de flúor, escovação supervisionada, orientações a respeito da higiene bucal e cuidados com próteses, dentre outros, tornam-se empecilhos, levando à piora da saúde bucal do paciente idoso e, consequentemente, de sua qualidade de vida, situação agravada em casos de idosos com alguma síndrome demencial (BIZERRIL D, et al., 2015; WARMLING AMF, et al., 2016). Para se alcançar a excelência no atendimento a este grupo populacional, é necessário que haja comprometimento não só dos profissionais como dos cuidadores, para que a higiene seja realizada de forma adequada até mesmo em pacientes que não possuam habilidade para executá-la sozinha e, assim, evitar/prevenir enfermidades orais e aprimorar a qualidade de vida destes clientes.

Sabe-se que idosos saudáveis e com síndromes demenciais possuem diferentes graus de dependência para realizar atividades diárias. A Doença de Parkinson (DP), por exemplo, causa tremores, distúrbios de locomoção e movimentos reduzidos e, assim, compromete a adequada execução da higiene bucal de seus portadores. Idosos acometidos por tal enfermidade podem apresentar xerostomia, sialorreia, disfagia e habilidade limitada para executar a higienização oral, o que reduz significativamente a qualidade de vida destes pacientes (PRADEEP AR, et al., 2015; WANG TF, et al., 2015).

Considerando-se os possíveis tratamentos para candidíase bucal, cárie radicular e xerostomia na população idosa, Silva HPR, et al. (2017) demonstraram não haver um padrão. As opções eram variadas e incluíam uso de medicamentos, desinfecção de próteses por diferentes técnicas, bochechos com solução de clorexidina e instrução de higiene bucal associada, ou não, a outros fármacos, e massagem funcional das glândulas salivares e músculos associados. Cabe ressaltar que esses tratamentos devem ser analisados criteriosamente com base na alteração bucal apresentada pelo paciente idoso e no seu estado de saúde/dependência. 
Os idosos também tendem a criar percepções próprias sobre a concepção de saúde bucal adequada. Uma pesquisa feita com 19 idosos saudáveis revelou que atribuem os seguintes significados a uma boa saúde bucal: ter uma boca limpa, ter boa saúde geral, ter um sorriso bonito, e se sentir bem com a condição bucal (BULGARELLI AF, et al., 2020). Desta forma, percebe-se que há uma relação entre uma boa autoestima e uma condição oral adequada, o que torna o idoso mais participativo na sociedade, aumentando sua rede de suporte social, o que, por conseguinte, irá auxiliá-lo no caso de se tornar dependente.

O idoso brasileiro, muitas vezes, não possui fácil acesso ao sistema de saúde, e vive em condições precárias de vida, incluindo-se, aqui, aqueles que vivem em comunidades de difícil acesso (LIMA RRT, et al., 2018). O estudo de Gontijo CF, et al. (2019) revelou a importância da coesão, suporte e participação social na vida de idosos, estando, inclusive estatisticamente associados com a condição de saúde do idoso (GONTIJO CF, et al., 2019). Assim, o idoso inserido em um contexto social adequado, com uma boa rede de suporte, também tenderá a apresentar melhores condições de saúde bucal, uma vez que, independentemente de realizar a higiene oral sozinho, ou não, haverá pessoas que irão auxiliá-lo em tal tarefa.

O cuidador de idosos é responsável pela atenção ao idoso, auxiliando-o com atividades diárias, o que inclui a realização do cuidado bucal (ALVES AKTM, 2018). Assim, uma forma de se alcançar uma higiene bucal adequada em ambiente domiciliar é a participação de cuidadores em eventos de educação em saúde, empregando estratégias de saúde bucal eficazes a fim se obter melhorias significativas na saúde oral de tais clientes. O cuidado com próteses e dentaduras também deve ser abordado, uma vez que se trata de uma clientela que, muitas vezes, faz uso de tais artigos. As dentaduras, por exemplo, devem ser trocadas a cada quatro ou cinco anos, porém existem pacientes que, por falta de orientação profissional, acabam usando-as por mais de 10 anos (WANG TF, et al., 2015; OSMARI D, et al., 2016). Ressalta-se que foram incluídos estudos que não constavam na amostra desta revisão integrativa para que conhecimentos correlacionados fossem abordados, abordando a discussão acerca do tema, além de revelar a escassez de trabalhos e a necessidade de se realizar mais estudos sobre a temática.

\section{CONCLUSÃO}

Os idosos são um grupo populacional que requer atenção especial quanto à sua saúde, incluindo, neste aspecto, a saúde bucal. Pacientes idosos com alguma síndrome demencial apresentam debilidades que dificultam e/ou impedem a execução de uma adequada higiene bucal. Neste âmbito, incluem-se os profissionais da saúde, que devem orientar os cuidadores e os próprios pacientes, quando possível, para a execução apropriada da higiene oral. Os trabalhos aqui incluídos levantaram a carência de informações e orientações repassadas pelos profissionais como um problema enfrentado por cuidadores. Dessa forma, torna-se essencial a educação permanente destes profissionais para que possam instruir seus pacientes de maneira eficaz e compreensível. Analisar as condições socioeconômicas, de saúde e hábitos de vida de cada cliente para direcionar as orientações também é de extrema importância para que sejam eficientes. Este estudo teve como limitações o fato de ser uma revisão sistemática e da pequena amostra de trabalhos recuperados, o que leva à necessidade de desenvolvimento de novas pesquisas acerca do tema abordado a fim de subsidiar políticas e programas de orientação e promoção da saúde bucal de qualidade em idosos, guiado pelos preceitos do Sistema Único de Saúde: universalidade, equidade e integralidade.

\section{REFERÊNCIAS}

1. ALVES AKTM, et al. Ações desenvolvidas por cuidadores de idosos institucionalizados no Brasil. Av. enferm. 2018; 36(3): 273-282.

2. ATENÇÃO DOMICILIAR. 2017. In: MINISTÉRIO da Saúde. Disponível em: https://www.saude.gov.br/acoes-eprogramas/melhor-em-casa-servico-de-atencao-domiciliar/atencao-domiciliar. Acesso em: 13 fev. 2020.

3. BARBE AG, et al. Self-assessment of oral health, dental health care and oral health-related quality of life among Parkinson's disease patients. Gerodontology. 2017; 34(1):135-43.

4. BIZERRIL DO, et al. Papel do cirurgião-dentista nas visitas domiciliares: atenção em saúde bucal. Rev Bras Med Fam Comunidade. 2016; 10(37): 1-8. 
5. BULGARELLI AF, et al. Uma perspectiva pós-moderna sobre a saúde bucal de idosos. Ciênc. saúde coletiva. 2020; 25(2): 741-748.

6. COLUSSI CF, CALVO MCM. Modelo de avaliação da saúde bucal na atenção básica. Cadernos de Saúde Pública, $2011 ; 27(9): 1731-1745$.

7. DUTRA CESV, SANCHEZ HF. Organização da atenção à saúde bucal prestada ao idoso nas equipes de saúde bucal da Estratégia Saúde da Família. Revista Brasileira de Geriatria e Gerontologia, 2015; 18(1): 179-188.

8. LIMA RRT, et al. Identificando necessidades e possíveis soluções: com a palavra, pessoas idosas na Atenção Primária à Saúde. Saúde debate. 2018; 42(119): 977-989.

9. MENDES KDS, et al. Revisão integrativa: método de pesquisa para a incorporação de evidências na saúde e na enfermagem. Texto e Contexto - Enfermagem, 2008; 17(4): 758-64.

10. MINISTÉRIO DA SAÚDE. Secretaria de atenção à saúde. Caderno de atenção básica, no 17. Disponível em: http://bvsms.saude.gov.br/bvs/publicacoes/saude_bucal.pdf. Acesso em: 11 abr. 2019.

11. MIRANDA GMD, MENDES ACG, SILVA ALA. O envelhecimento populacional brasileiro: desafios e consequências sociais atuais e futuras. Rev bras geriatr gerontol, 2016; 19(3): 507-519.

12. MOREIRA RM, et al. Qualidade de vida, saúde e política pública de idosos no Brasil: uma reflexão teórica. Kairós Gerontologia, 2013; 16(2): 27-38.

13. OSMARI D, et al. Behaviour of the elderly with regard to hygiene procedures for and maintenance of removable dentures. Oral health and preventive dentistry, 2016; 14(1): 21-6.

14. PRADEEP AR, et al. Clinical evaluation of the periodontal health condition and oral health awareness in Parkinson's disease patients. Gerodontology, 2015; 32(2): 100-6.

15. SALES MVG, et al. Condições de saúde bucal do idoso no Brasil: uma revisão de literatura. Arch Health Invest, 2017; 6(3): 120-124.

16. SANTOS CS, et al. Fatores asociados à demência em idosos. Ciênc. saúde coletiva. 2020; 25(2): 603-611.

17. SILVA HPR, et al. Abordagem das afecções bucais mais prevalentes em idosos: uma revisão integrativa com foco na atenção primária. Revista Brasileira de Geriatria e Gerontologia, 2017; 20(3): 432-443.

18. SIMÕES ACA, CARVALHO DM. A realidade da saúde bucal do idoso no Sudeste brasileiro. Ciência e Saúde Coletiva, 2011; 16(6): 2975-2982.

19. SOUZA AC, et al. Contextualizando a estratégia saúde da família na atenção primária em saúde. In: ABRAHÃO AL, et al. Estratégia saúde da família: desafios e novas possibilidades para a atenção básica em saúde. Niterói: EDUFF; $2012 ; 24-48$.

20. WANG TF, et al. Effect of oral health education programs for caregivers on oral hygiene of the elderly: A systemic review and meta-analysis. Int J Nurs Stud, 2015; 52(6):1090-6.

21. WARMLING AMF, et al. Home-based oral health care strategies of elderly people with Alzheimer's disease. Revista Brasileira de Geriatria e Gerontologia, 2016; 19(5): 851-60. 